\title{
Tempo de reação motora no diabetes mellitus tipo 2: revisão integrativa da literatura
}

\author{
Motor reaction time in type 2 diabetes: an integrative review of the literature \\ Tiempo de reacción motor en diabetes mellitus tipo 2: revisión integrativa de la literatura
}

Recebido: 09/08/2021 | Revisado: 13/08/2021 | Aceito: 15/08/2021 | Publicado: 17/08/2021

\author{
Flávia Martins Gervásio \\ ORCID: https://orcid.org/0000-0003-1270-1608 \\ Universidade Estadual de Goiás, Brasil \\ E-mail: flavia.gervasio@hotmail.com \\ Aline Helena Nascimento Veloso \\ ORCID: https://orcid.org/0000-0001-7329-1986 \\ Universidade Estadual de Goiás, Brasil \\ E-mail: aline.veloso@ aluno.ueg.br \\ Luíza Rodrigues de Assis Brito \\ ORCID: https://orcid.org/0000-0003-1949-7726 \\ Universidade Estadual de Goiás, Brasil \\ E-mail: luiza@aluno.ueg.br \\ Alexandre Barroso de Farias \\ ORCID: https://orcid.org/0000-0001-7304-9866 \\ Universidade Federal Fluminense, Brasil \\ E-mail: alexandref@id.uff.br \\ Franassis Barbosa de Oliveira \\ ORCID: https://orcid.org/0000-0001-5145-1491 \\ Universidade Estadual de Goiás, Brasil \\ E-mail: franassis_oliveira@yahoo.com.br
}

\begin{abstract}
Resumo
Altos níveis glicêmicos prejudicam principalmente o sistema nervoso periférico, causando danos sensitivos e motores. Medidas neuromotoras de tempo de reação (TR) mostraram-se um eficiente indicador de prejuízo funcional em diferentes populações. O presente estudo objetivou identificar e analisar nas literaturas nacional e internacional o comportamento do TR no diabetes mellitus 2 (DM2) e os principais fatores intervenientes. Trata-se de uma revisão integrativa, conduzida nas bases de dados LILACS, BIREME, SCOPUS, Cochrane e Pubmed no período de julho e agosto de 2020. Ao final foram selecionados 14 artigos todos na língua inglesa. Os resultados mostram que o TR no DM2 associado à neuropatia foi significativamente maior em comparação a outras populações. Portanto, a medida do TR é uma alternativa viável no diagnóstico das disfunções neurológicas do DM2, uma vez que se mostrou sensível à mudança da velocidade dos impulsos nervosos, mostrando-se lentificada. Esta avaliação pode ser aplicada como medida diagnóstica e preventiva no tratamento destes pacientes, com efeito aos casos graves como úlceras e amputações, triados previamente. Estudos futuros podem investigar possível padronização instrumental e metodológica, além da validação de um produto nacional que explore o TR na população brasileira.
\end{abstract} Palavras-chave: Tempo de reação; Diabetes mellitus; Diabetes mellitus tipo 2.

\begin{abstract}
High blood glucose levels results in deleterious effects to the the peripheral nervous system, causing sensory and motor damage. Neuromotor reaction time (RT) measurements are a sensitive indicator of functional impairment in different populations. The aim of the present study was to identify and analyze national and international literature on the impact of diabetes mellitus 2 (DM2) over RT measurements and the main mechanisms for such correlation. This integrative review was conducted by ressarching the LILACS, BIREME, SCOPUS, Cochrane and Pubmed databases in July 2020 and August 2020. In the end, 14 articles were selected, all in English. The results show that the TR in DM2 cases associated with neuropathy was higher than expected when compared to other populations. Therefore, the RT measurement is a viable alternative in the diagnosis of DM2-associated neurological disorders, due do its sensitivity to changes in the velocity of nerve impulse conduction, slowed down in DM-2 associated neuropathy. RT assessments can be employed as diagnostic and screening measures when dealing with these patients, with emphasis on preventing severe outcomes such as ulcers and amputations. Future studies should investigate possible instrumental and methodological standardization, in addition to the validation of a national product that explores RT in the Brazilian population.
\end{abstract}

Keywords: Reaction time; Diabetes mellitus; Type 2 diabetes mellitus.

\section{Resumen}

Los niveles elevados de glucosa en sangre afectan principalmente al sistema nervioso periférico, provocando daños sensoriales y motores. Las mediciones del tiempo de reacción neuromotora (RT) demostraron ser un indicador 
eficiente del deterioro funcional en diferentes poblaciones. Este estudio tuvo como objetivo identificar y analizar en la literatura nacional e internacional el comportamiento de la RT en la diabetes mellitus 2 (DM2) y los principales factores intervinientes. Se trata de una revisión integradora, realizada en las bases de datos LILACS, BIREME, SCOPUS, Cochrane y Pubmed en el período de julio y agosto de 2020. Al final, se seleccionaron 14 artículos, todos en inglés. Los resultados muestran que la RT en DM2 asociada a neuropatía fue significativamente mayor en comparación con otras poblaciones. Por lo tanto, la medición de la RT es una alternativa viable en el diagnóstico de los trastornos neurológicos de la DM2, ya que fue sensible a los cambios en la velocidad de los impulsos nerviosos, siendo enlentecidos. Esta valoración puede aplicarse como medida diagnóstica y preventiva en el tratamiento de estos pacientes, con efecto en casos graves como úlceras y amputaciones, previamente cribados. Los estudios futuros pueden investigar la posible estandarización instrumental y metodológica, además de la validación de un producto nacional que explora el RT en la población brasileña.

Palabras clave: Tiempo de reacción; Diabetes mellitus; Diabetes mellitus tipo 2.

\section{Introdução}

Diabetes mellitus é uma doença crônica caracterizada pela hiperglicemia relacionada à secreção de insulina insuficiente, ação defeituosa da insulina ou ambos (American Diabetes Association, 2012). No diabetes mellitus tipo 2 (DM2), o indivíduo se torna resistente à insulina ou incapaz de produzi-la suficientemente. É um problema de saúde global e sério, sendo o tipo mais comum, correspondente a $90 \%$ a $95 \%$ dos casos. Atinge em maiores proporções a população de renda baixa e média e tem relação com as mudanças sociais, econômicas e culturais, o envelhecimento, o estilo de vida com padrões de comportamento não saudáveis, com redução da atividade física, mudanças na dieta que incluem maior consumo de alimentos processados e açúcar, obesidade, processo de urbanização crescente e não planejado, além da desnutrição fetal e aumento da exposição de hiperglicemia ao feto durante o período gestacional. Apesar de ser mais comum em adultos, a DM2 tem crescido entre crianças e adolescentes também (World Health Organization, 2016, 2019).

Primeiramente, cerca de 422 milhões de pessoas em todo o mundo têm diabetes e a maioria vive em países de baixa e média renda (World Health Organization, 2020). Segundo boletim epidemiológico divulgado pelo Ministério da Saúde, a prevalência de Diabetes Mellitus no Brasil passou de 5,5\% em 2006 para 7,4\% em 2019, um aumento relativo de 34,5\% no período, sendo mais prevalente entre mulheres e adultos acima dos 65 anos (Ministério da Saúde do Brasil, 2020).

Os altos níveis glicêmicos prejudicam o sistema neurológico, sobretudo o periférico, com danos sensitivos e motores, caracterizada pela neuropatia periférica. Equipamentos capazes de indicar alterações neuromotoras em pacientes com diabetes são importantes ferramentas clínicas de diagnóstico precoce bem como da extensão do dano (Sociedade Brasileira de Diabetes et al., 2017).

As medidas neuromotoras do tempo de reação (TR), intervalo entre o aparecimento do estímulo e a resposta, tem se mostrado um eficiente indicador de prejuízos funcionais em idosos, atletas e doenças como o Alzheimer (Pageaux \& Lepers, 2018). Entretanto, os relatos na DM2, ainda não apresentam consenso sobre a presença da alteração no TR e/ou seus efeitos nas funções neuromotoras destes pacientes. Diante do exposto, esse trabalho tem por objetivo identificar e analisar nas literaturas nacional e internacional o comportamento do TR motor no DM2 e os principais fatores intervenientes.

O interesse pelo tempo de reação em pessoas com diabetes data desde a década de 60, quando Dobrzański e Rychta (1968) propuseram que o tempo de reação visual desses pacientes seria o dobro do encontrado em indivíduos saudáveis. Desde então, essa questão foi estudada em diversos contextos e causas, ultrapassando a investigação puramente em saúde. Devido ao aumento da população atingida por essa doença metabólica e as variadas implicações funcionais dessa resposta lentificada, percebe-se desenvolvimento de tecnologias capazes de prever, não somente os graus de comprometimento da condução dos estímulos, mas também como atividades de vida diária podem ser afetadas nesse contexto, como dirigir, por exemplo.

Ao longo dos anos a investigação seguiu um raciocínio voltado à aplicação prática. Richerson et al. (2005) pautaram sua pesquisa nos achados primários puramente transversais, os quais já propunham um aumento no TR para pacientes com diabetes. No entanto, seu estudo foi referência para a investigação dessa variável na última década, uma vez que incluiu novas 
modalidades de TR e procurou correlacioná-las com a funcionalidade, idade, aumento no número de quedas e diminuição da sensibilidade. Com seus resultados aumentados para o toque plantar, movimento de corpo inteiro e reação auditiva, outros pesquisadores como Sanchez-Marin e Padilla-Medina (2010), aprofundaram esses achados para o contexto do tempo de reação à mudança de cores no semáforo. Estas pesquisas sinalizam a importância do estudo do tempo motor no diabético e seu impacto, atingindo a análise da segurança, nas atividades de vida diária.

A neuropatia periférica é discutida como potencial fator primário nos aumentos de TR, já que está associada à degeneração axonal e à desmielinização, fato que leva à lentificação da velocidade de condução sensorial mesmo em pacientes com diabetes com alteraçães leves ao teste dos monofilamentos e com duração menor que 10 anos da doença. Isso pode sugerir que testes como o de reação ao toque plantar são parâmetros úteis no diagnóstico precoce e na determinação da extensão de seus danos periféricos (Richerson et al., 2005).

A caracterização clínica e os impactos orgânicos dos altos níveis glicêmicos no organismo são amplamente descritos (World Health Organization \& International Diabetes Federal, 2006). Entretanto, o comportamento do tempo de reação motora por meio das ações da mão ou do pé, frente a um estímulo visual ou auditivo, bem como, suas implicações em relação a idade, cronicidade da doença, tipos de reação mais investigadas e afetadas e sua relação com as funções cognitivas, laboral ou impacto funcional ainda são claros na literatura vigente. Diante do supracitado, esta revisão tem por objetivo identificar e analisar os fatores relacionados ao tempo de reação motora simples e de escolha em adultos com DM2, além de discorrer sobre o seu comportamento e os fatores intervenientes.

\section{Metodologia}

Trata-se de uma revisão integrativa da literatura, ou seja, uma pesquisa bibliográfica que permitiu levantar as principais informações teóricas sobre o tema, desenvolvida por meio de seis etapas que se fundamentadas na Prática Baseada em Evidências, conforme proposto por Botelho, Cunha e Macedo (2011).

A primeira etapa consistiu na identificação do tema e levantamento da pergunta norteadora. A busca do referencial do estudo adotou o instrumento evidence-based practice, em que a questão norteadora partiu do anagrama PICO (representando problema, intervenção, controle e outcome, palavra da língua inglesa significando desfecho). Com P: Diabetes tipo 2. I: medida do tempo de reação C: indivíduo saudável/hígido O: fatores que interferem no tempo de reação, a fim de responder a seguinte pergunta norteadora: Qual o comportamento do tempo de reação motora nas pessoas com DM2 e quais os fatores intervenientes?

A segunda etapa consistiu no estabelecimento de critérios de inclusão e exclusão. Os critérios de inclusão foram artigos completos nos idiomas inglês, português ou espanhol; publicados entre os anos de 2005 e 2020, realizados em humanos com diabetes mellitus tipo 2, sob tratamento medicamentoso, cuja a obtenção do tempo motor adotasse metodologia instrumental. As buscas consideraram as bases de dados PubMed, Centro Latino-Americano e do Caribe de Informação em Ciências da Saúde (BIREME), Cochrane e SCOPUS. A escolha das bases de dados se deu devido ao quantitativo de indexação de artigos da área da saúde, e teve como finalidade ampliar o escopo da pesquisa e minimizar possíveis vieses. Foram excluídos os artigos não-primários, como os de relato de opinião e as revisões; teses e dissertações. Pessoas com histórico de diabetes associados a outros distúrbios neurológicos que influenciam o tempo motor como lesões desmielinizantes, espásticas, entre outros, além de artigos em que os pacientes possuíam outras doenças neurológicas, doenças reumáticas e ortopédicas, condições auditivas e visuais alteradas, como baixa visão, retinopatias e outras também foram excluídos.

A terceira etapa consistiu na busca dos estudos primários nas bases de dados. Para isso, os descritores controlados selecionados do Medical Subject Headings (MeSH) foram diabetes, diabetes mellitus, diabetics, type 2 diabetes, reaction time, response time e dos Descritores em Ciências da Saúde (DecS) foram: diabetes, diabetes mellitus, diabéticos/diabetics, 
tempo de reaçãol tiempo de reacción, tempo de resposta/tiempo de respuesta, além de outros termos como motor reaction time/tempo de reação motora/ tiempo de reacción del motor, motor reaction/reação motora/ reacción motora, simple reaction time/tempo de reação simples/tiempo de reacción simple, choice reaction time/tempo de reação de escolha/tiempo de reacción de elección. Adotou-se o operador booleano "AND" para combinar os termos entre si e "NOT" como forma de delimitar a busca, excluindo os termos retinopathy, stroke, traumatic brain injury, low vision, vision disorders e hearing loss.

A quarta etapa consistiu na extração de dados, com seleção dos artigos de acordo com a leitura do título e resumo. A quinta etapa foi a avaliação dos estudos selecionados realizada por meio da leitura na íntegra dos artigos. A sexta etapa foi a interpretação para obtenção dos resultados e apresentação da revisão.

O fluxograma a seguir apresenta o resultado de seleção de artigos segundo os critérios do estudo (Figura 1).

Figura 1. Fluxograma das etapas para selecionar os artigos da revisão.

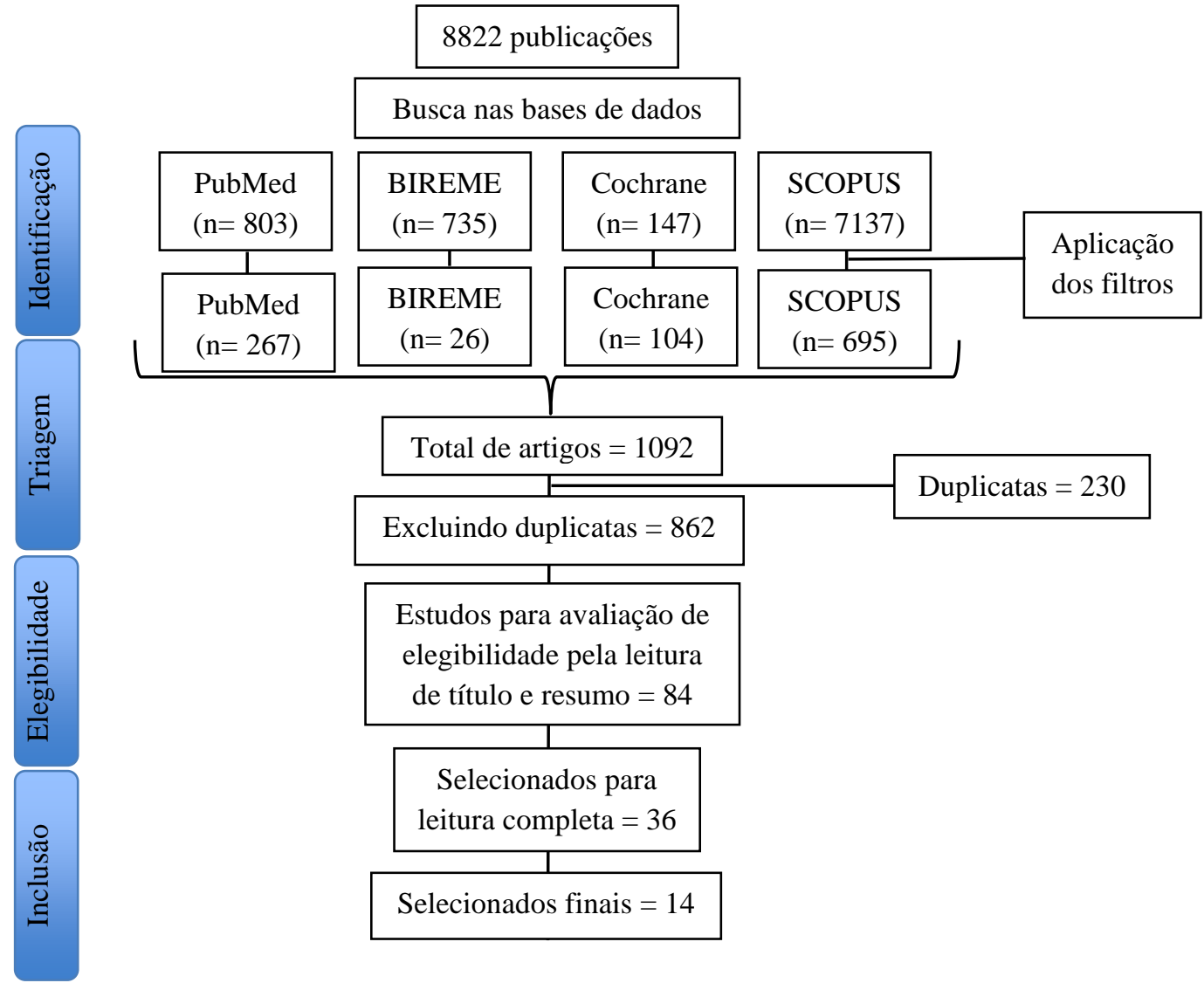

Fonte: Autores (2021).

\section{Resultados}

Foram incluídos no estudo 14 artigos. Estes foram dispostos em quadro sinóptico (Figura 1) em ordem cronológica de publicação, de modo a apresentar público estudado, faixa etária, equipamentos utilizados na coleta no tempo de reação motora e principais desfechos.

No que tange ao ano de publicação, em 2017 foram publicados 4 artigos, em 2014 e 2015 foram publicados 2 em cada ano e em 2005, 2010, 2011, 2016, 2019 e 2020 somente 1 artigo cada, todos em inglês. Com relação ao país-sede do estudo, 5 foram desenvolvidos nos Estados Unidos e 4 na Índia. Espanha, China, Canadá, Inglaterra e México apresentaram apenas 1 artigo cada. 
Na identificação dos diferentes tempos de reação, em 6 (seis) artigos os autores apresentaram o conceito de cada tipo de tempo de reação avaliado e nos demais essa informação não está presente. No todo, 9 (nove) investigaram o tempo de reação visual simples, 6 (seis) estudaram o tempo de reação visual de escolha, 4 (quatro) trataram do tempo de reação auditivo simples, 4 (quatro) mensuraram o tempo de reação ao freio, 2 (dois) se voltaram a tempos de reação simples de corpo inteiro, 1 (um) pesquisou o tempo de reação de escolha de corpo inteiro e em 1 (um) estudo, cuidou-se do tempo de reação ao toque plantar. Foram utilizados 16 instrumentos diferentes para mensurar o tempo de reação nos estudos revisados, sendo que, para 5 foi justificada a escolha dos mesmos e para 11 não; 2 utilizaram instrumentos desenvolvidos pela própria instituição ou em desenvolvimento por outras instituições e 8, equipamentos disponíveis no mercado. Em 6 dos estudos revisados, não foi detalhada a proveniência e o modelo do equipamento empregado na medida do tempo de reação motor.

Evidenciou-se um estudo coorte, três ensaios clínicos randomizados controlados e 10 estudos transversais. Em relação ao tipo de revista nas quais foram publicados os artigos incluídos na revisão, 3 foram publicados em revistas de Medicina em geral e 3 em revistas de Reumatologia, enquanto os demais nas áreas da Engenharia Biomédica, Biologia Computacional, Neuropsicologia, Biologia Molecular, Biogerontologia, Fisiatria e Reabilitação, Fisiologia e Farmacologia e Segurança no Trânsito, com 1 artigo em cada área. Os resultados da síntese dos artigos selecionados são apresentados no Quadro 1.

Quadro 1. Quadro com caracterização dos indicadores bibliométricos dos estudos selecionados para a revisão integrativa.

\begin{tabular}{|c|c|c|c|c|c|}
\hline Autores & Amostral: quantidade & $\begin{array}{l}\text { Idade } \\
\text { média } \\
\text { (anos) }\end{array}$ & $\begin{array}{l}\text { Design do } \\
\text { estudo }\end{array}$ & Principais resultados & $\begin{array}{l}\text { Fatores de risco } \\
\text { relacionados }\end{array}$ \\
\hline $\begin{array}{l}\text { Richerson et al., } \\
2005\end{array}$ & $\begin{array}{l}\text { DM2: } 13 \\
\text { Controle: } 24\end{array}$ & 60 & Transversal & $\begin{array}{l}\text { TRTP e TRCI maiores em DM2 } \\
(p<0.05) . \text { TRAS não diferiu } \\
\text { entre grupos }\end{array}$ & Neuropatia periférica \\
\hline $\begin{array}{l}\text { Sanchez-Marin \& } \\
\text { Padilla-Medina, } \\
2010\end{array}$ & $\begin{array}{l}\text { DM2: } 20 \\
\text { Controle: } 20\end{array}$ & 46 & Transversal & $\begin{array}{l}\text { TRVS médio e TRVE } \\
\text { aumentados no grupo DM2 }\end{array}$ & $\begin{array}{l}\text { Idade, duração do diabetes } \\
\text { e controle glicêmico }\end{array}$ \\
\hline $\begin{array}{l}\text { Whitehead et al., } \\
2011\end{array}$ & $\begin{array}{l}\text { DM2: } 41 \\
\text { Controle: } 424\end{array}$ & 71,5 & $\begin{array}{l}\text { Ensaio clínico } \\
\text { randomizado }\end{array}$ & $\begin{array}{l}\text { DM2 mais lento que os } \\
\text { controles em TRVS e TRVE (p } \\
<.05)\end{array}$ & 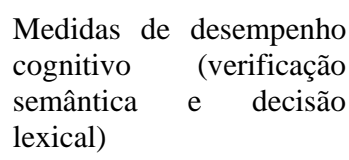 \\
\hline Muhil, 2014 & $\begin{array}{l}\text { DM2 HbA1c }<7,0: 100 \\
\text { DM2 HbA1c }> \\
\text { 7,0:100 } \\
\text { Controle: } 100\end{array}$ & 50 & Transversal & $\begin{array}{l}\text { Para DM2 HbA1C }>7 \\
\text { apresentou o maior aumento no } \\
\text { TRAS e TRVS }(p \leq 0.01)\end{array}$ & Controle glicêmico \\
\hline $\begin{array}{l}\text { Gilchris et al., } \\
2014\end{array}$ & DM2: 27 & 67 & $\begin{array}{l}\text { Ensaio clínico } \\
\text { randomizado }\end{array}$ & $\begin{array}{l}\text { Após } 2 \text { semanas de suco de } \\
\text { beterraba, TRVS mais rápido no } \\
\text { grupo suco de beterraba em } \\
\text { relação ao grupo placebo ( } \mathrm{p}= \\
0.009 \text { ) }\end{array}$ & $\begin{array}{l}\text { Fatores nutricionais } \\
\text { (óxido nítrico) }\end{array}$ \\
\hline Khode et al., 2015 & $\begin{array}{l}\text { DM2: } 60 \\
\text { Controles: } 60\end{array}$ & 50 & Transversal & $\begin{array}{l}\text { TRVS }(p=0.007) \text { e TRVE } \\
(p=0.020) \text { atrasados em DM2, } \\
\text { assim como TRSCI }(p=0.001) \text { e } \\
\text { TRECI }(p=0.003)\end{array}$ & Neuropatia periférica \\
\hline $\begin{array}{l}\text { Mungal et al., } \\
2015\end{array}$ & $\begin{array}{l}\text { DM2: } 50 \\
\text { Controles: } 50\end{array}$ & 48 & Transversal & $\begin{array}{l}\text { TR audiovisual aumentado em } \\
\text { DM2 }(\mathrm{p}<0.05)\end{array}$ & Neuropatia periférica \\
\hline
\end{tabular}




\begin{tabular}{|c|c|c|c|c|c|}
\hline $\begin{array}{l}\text { Redondo et al., } \\
2016\end{array}$ & $\begin{array}{l}\text { DA: } 22 \\
\text { DM2: } 20 \\
\text { Controles: } 23\end{array}$ & 74,5 & Transversal & $\begin{array}{l}\text { Idosos com DA apresentaram } \\
\text { TRs mais lentos que saudáveis } \\
(\mathrm{p}<0.05) \text {, mas não diferiram dos } \\
\text { DM2 (p>0.05). TR dos DM2 } \\
\text { não diferiu dos saudáveis } \\
(\mathrm{p}>0.05)\end{array}$ & $\begin{array}{l}\text { Idade, acometimentos } \\
\text { neurológicos como a DA }\end{array}$ \\
\hline $\begin{array}{l}\text { Richardson et al., } \\
2017\end{array}$ & $\begin{array}{l}\text { DM2 com neuropatia: } \\
26 \\
\text { DM2 sem neuropatia: } \\
16\end{array}$ & 69 & $\begin{array}{l}\text { Longitudinal de } \\
\text { coorte } \\
\text { prospectivo }\end{array}$ & $\begin{array}{l}\text { Em DM2 com neuropatia, a } \\
\text { razão entre TRVE e o TRVS } \\
\text { mais rápido associados com } \\
\text { tempo de apoio unipodal mais } \\
\text { longo e a variabilidade e } \\
\text { intervalo da largura do passo } \\
\text { diminuída }\end{array}$ & Neuropatia periférica \\
\hline $\begin{array}{l}\text { Meyr \& Spiess, } \\
2017\end{array}$ & $\begin{array}{l}\text { DM2: } 25 \\
\text { Controles: } 25\end{array}$ & 32,7 & Transversal & $\begin{array}{l}\text { Para DM2, TRF } 37,89 \% \text { mais } \\
\text { lento }(p<0,001) \text {, acima do limite } \\
\text { de segurança }(0,70 \text { segundos })\end{array}$ & $\begin{array}{l}\text { Neuropatia periférica, } \\
\text { segurança no trânsito }\end{array}$ \\
\hline Spiess et al., 2017 & $\begin{array}{l}\text { DM2 com neuropatia: } \\
25 \\
\text { DM2 sem neuropatia: } \\
25\end{array}$ & 51,5 & Transversal & 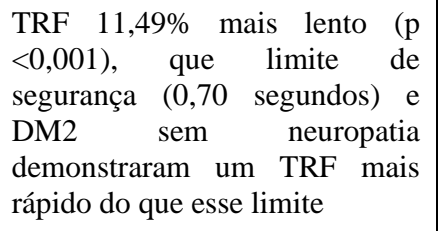 & $\begin{array}{l}\text { Neuropatia periférica, } \\
\text { segurança no trânsito }\end{array}$ \\
\hline $\begin{array}{l}\text { Sansosti et al., } \\
2017\end{array}$ & $\begin{array}{l}\text { DM2 com neuropatia } \\
\text { e histórico de } \\
\text { patologia de pé } \\
\text { diabético: } 20 \\
\text { DM2 sem histórico de } \\
\text { patologia de pé } \\
\text { diabético: } 20\end{array}$ & 58,65 & Transversal & $\begin{array}{l}\text { Motoristas sem história de } \\
\text { patologia específica no pé } \\
\text { demonstraram TRF médio } \\
11,11 \% \text { mais lento }(p<0,001) \text {. } \\
\text { Ambos os grupos demonstraram } \\
\text { TRF médio mais lento do que o } \\
\text { limite sugerido de } 0,70 \\
\text { segundos }\end{array}$ & $\begin{array}{l}\text { Neuropatia periférica, pé } \\
\text { diabético, segurança no } \\
\text { trânsito }\end{array}$ \\
\hline Kour et al., 2019 & $\begin{array}{l}\text { DM2: } 148 \\
\text { Controles: } 74\end{array}$ & 32,5 & $\begin{array}{l}\text { Ensaio clínico } \\
\text { randomizado }\end{array}$ & $\begin{array}{l}\text { Resistência cardiorrespiratória } \\
\text { com melhora significativa em } \\
\text { seis meses }(\mathrm{p}<0,001) \text {. DM2 } \\
\text { tiveram velocidade de condução } \\
\text { nervosa e TRVS diminuídos em } \\
\text { comparação com controles (p } \\
<0,001) \text {. TRAS: nenhuma } \\
\text { diferença significativa }\end{array}$ & $\begin{array}{l}\text { Neuropatia periférica, } \\
\text { controle glicêmico, } \\
\text { parêmetros de resistência } \\
\text { cardiorrespiratória (índice } \\
\text { de aptidão física } \\
\text { VO2 máximo) }\end{array}$ \\
\hline Ma et al., 2020 & $\begin{array}{l}\text { DM2: } 27 \\
\text { Controles: } 29\end{array}$ & 50 & Transversal & $\begin{array}{l}\text { DM2 tiveram pior desempenho } \\
\text { de direção, com maior desvio da } \\
\text { linha central }(p=0,006) \text {, maior } \\
\text { TRF }(p=0,001) \text { e menor tempo } \\
\text { mínimo para colisão }(p=0,002)\end{array}$ & $\begin{array}{l}\text { Neuropatia periférica, } \\
\text { segurança no trânsito }\end{array}$ \\
\hline
\end{tabular}

Legenda: DM2: diabetes melitus tipo 2. TRVS: Tempo de reação visual simples. TRVE: Tempo de reação visual de escolha. TRAS: Tempo de reação auditivo simples. TRAE: Tempo de reação auditivo de escolha. TRF: Tempo de resposta ao freio. TRSCI: Tempo de reação simples de corpo inteiro. TRECI: Tempo de reação de escolha de corpo inteiro. MMSS: Membros Superiores. MMII: Membros inferiores. TRTP: Tempo de reação ao toque plantar. DA: Doença de Alzheimer. Fonte: Autores (2021).

\section{Discussão}

Identificou-se que os tempos de reação motora em pacientes com diabetes em despeito a idade, cronicidade da doença ou associação com neuropatia diabética foram mais lentos em comparação com pessoas hígidas. Estes tempos aumentam sua latência na associação com respostas cognitivas, ou seja, nas tarefas que envolvem escolha. O aumento dos tempos de reação esteve presente, nas respostas visual, auditiva de membros superiores, de freio ou toque plantar nos membros inferiores e de corpo inteiro. 
Um aspecto relevante é que não há a padronização de um espectro etário para a investigação do tempo de reação no diabetes, porém predominam grupos compostos por adultos mais velhos. Isso pode estar relacionado com a maior prevalência do diabetes tipo 2 como comorbidade nessa população, visto que essa apresenta alto risco de desenvolver a doença devido aos efeitos combinados do aumento da resistência à insulina, associada à adiposidade, sarcopenia e inatividade física, e do comprometimento da função das ilhotas pancreáticas com o envelhecimento (Kirkman et al., 2012).

Quanto à avaliação glicêmica nos estudos, percebeu-se o uso da hemoglobina glicada (HbA1c) como padrão. Os níveis de HbA1c são diretamente proporcionais à glicemia média dos últimos 2 a 3 meses e HbA1c > 7\% está associado a um risco progressivamente maior de complicações crônicas, visto que a hiperglicemia favorece a oxidação da glicose e a liberação de radicais livres como peroxinitrito, que prejudicam a transmissão do sinal nervoso e velocidade de condução (Sociedade Brasileira de Diabetes et al., 2017). Muhil (2014) afirma em seu estudo que há maior tempo de reação auditiva e visual atrasados em pacientes com DM2 sem controle glicêmico, o que relaciona-se aos maiores resultados para TRAS e TRVS. Cabe ressaltar que a HbA1c é amplamente empregada como método de investigação das taxas glicêmicas, porém, por ser um valor médio, uma grande variação da glicemia não é detectada por meio desse exame. A avaliação da variabilidade da glicemia, medida das flutuações da glicose, ou outros parâmetros relacionados à homeostase da glicose, durante um determinado intervalo de tempo, é muito relevante para esse perfil de pacientes (Ceriello et al., 2019), e ainda não foi investigado na literatura investigada.

Outro fator que pode estar atrelado ao tempo de reação é a duração do diabetes. Para Richerson et al. (2005), portadores de diabetes com menos de 10 anos de doença, apresentaram TR aumentado. Contudo, Ma et al. (2020) relata que a duração da doença não apresentou correlações significativas com qualquer um dos indicadores de desempenho investigados. Sanchez-Marin e Padilla-Medina (2010) também revelam que TRVS e duração do diabetes não apresentam correlação significativa. Porém, em relação à tomada de decisão clínica, os autores encontraram resultados que correlacionam consideravelmente o tempo decorrido desde o diagnóstico com o índice de desempenho. Importante destacar que é considerado como tempo de duração do diabetes o tempo decorrido desde o diagnóstico até o momento de realização do estudo e que há dificuldade para determinar o tempo exato de acometimento, uma vez que, comumente, a doença é assintomática ou oligossintomática, sendo o diagnóstico realizado por exames de rotina ou manifestações das complicações crônicas (Sociedade Brasileira de Diabetes et al., 2017).

O diabetes pode afetar as funções cognitivas (Almeida-Pititto et al., 2008). O decréscimo da taxa média no desempenho acelerado em todas as tarefas foi confirmado para indivíduos com DM2 (Khode et al., 2015; Whitehead et al., 2011) por meio de uma leitura cronoscópica, que distinguiu o efeito central em relação aos efeitos periféricos quando os TR são retardados, indicando que o TRECI pode ser preditivo de disfunção cognitiva em pacientes com diabetes. Redondo et al. (2016) constataram que pacientes com Doença de Alzheimer e DM2 apresentam prejuízos no funcionamento executivo e na memória de trabalho e que fatores de risco relacionados ao diabetes, como hipertensão, doenças cardiovasculares e síndrome metabólica podem ser responsáveis pelo comprometimento cognitivo e maiores chances de desenvolvimento de demências.

Segundo Ryan et al. (2016), a diminuição da velocidade de processamento cognitivo pode ser um elemento importante no perfil do paciente com diabetes tipo 2, que frequentemente manifesta déficits leves de aprendizado e memória. Essas mudanças cognitivas são acompanhadas por atrofia cortical significativa e anormalidades microestruturais de massa branca e cinzenta, relacionados à duração do diabetes e ao pior controle metabólico, resultando na redução da eficiência mental (Moheet et al., 2015).

As condições nutricionais podem afetar o processamento segundo Gilchrist et al. (2014), que testaram a hipótese de que a suplementação de nitrato na dieta aumentaria o desempenho cognitivo em pacientes com DM2, cujos resultados 
apresentaram melhora do TR. Isto seria explicado por mecanismos subjacentes como a melhora na perfusão cerebral e melhor acoplamento neurovascular.

O TRV é o mais investigado devido a facilidade no desenvolvimento de equipamentos para essa medida, principalmente por meio de monitores de computador acoplados a acessórios como teclados, mouses, pedais, dispositivos sensíveis ao toque e controles. Também contribui para isso a grande variedade de estímulos possíveis, a exemplo de círculos, setas, diferentes cores, testes psicofísicos e simuladores de direção, fato que dificulta a padronização dos estudos e mostrou concordância com os achados de Crocetta et al. (2014).

$\mathrm{O}$ diabetes parece afetar expressivamente as tarefas que envolvem o caminho visual e outros centros cerebrais que processam informações visuais. Devido ao envolvimento de muitas vias colaterais na via visual e à diferença no tipo de estimulação dos bastonetes e cones do que o órgão de Corti da orelha interna, o tempo de reação visual demonstrou resultados de maior comprometimento no tempo de reação em relação à auditiva (Muhil, 2014). O TRA apresentou certa controvérsia nos estudos encontrados, uma vez que, mesmo que a frequência sonora baixa não tenha apresentado diferença significativa, para as frequências média e alta foi encontrado aumento. Os tempos de resposta sonora foram menores em comparação às outras medidas de TR investigados nos indivíduos com diabetes, que segundo Richerson et al. (2005) deve-se ao fato de ser uma tarefa simples e de caminho curto entre o sistema nervoso periférico e central.

A identificação precoce de um tempo de reação aumentado pode ser uma forma valiosa de prevenção de quadros mais severos de complicações tardias do diabetes. Assim, a identificação e a adoção de estratégias terapêuticas que visem o melhor controle dessa doença podem prevenir condições degenerativas graves, como úlceras que não cicatrizam e que podem levar à amputação de membros e incapacidade. Esses aspectos geram impacto direto na qualidade de vida dos pacientes e a medida do TR é uma alternativa de baixo custo e fácil manuseio. Mungal et al. (2015) sugerem, ainda, que medidas de TR audiovisual sejam incluídas no teste de aptidão para profissionais (como motoristas, pilotos e médicos), em vista da relevância de um TR ideal na tomada decisões instantâneas e de alto risco. Os estudos sequenciais de Meyr e Spiess (2017), Spiess et al. (2017) e Sansoti et al. (2017) observaram o comportamento do diabético tipo 2 no trânsito, principalmente quanto ao TF, e concluíram que apresentam um TF aumentado em relação à aqueles sem diabetes, mas entre portador de diabetes com e sem histórico de pé diabético não há uma diferença significante.

A investigação mostrou-se importante quanto ao bom desenvolvimento de atividades de vida diária (AVD’S) nesse público e sua funcionalidade. O aumento do TR em pacientes com diabetes pode afetar a postura, a marcha e as sensações periféricas, fatores que se associam a maior probabilidade de queda. Richardson et al. (2017) demonstraram a proporção entre TRVE preciso e TRVS diminuído fortemente associada com tempo de apoio unipodal mais longo e diminuição da variabilidade e amplitude da largura do passo em uma superfície irregular. No estudo de Kour et al. (2019) houve uma diminuição no TRAS e no TRVS com seis meses de terapia intervencionista, o que indica melhora no desempenho sensóriomotor. Essas alterações podem estar relacionadas ao aumento da capacidade de processamento do sistema nervoso central com a terapia com exercícios aeróbicos e resistidos. Nesse contexto, estudos futuros podem aprofundar a investigação quanto às implicações funcionais de um TR aumentado, sobretudo quanto ao autocuidado e a execução de tarefas simples, além dos efeitos de um tratamento multidisciplinar mais específico.

O presente estudo identificou uma preocupação e propostas de ação integradas entre as áreas da saúde/biologia e da engenharia. Assim, a Medicina foi a área com maior número de estudos, porém não foram encontrados estudos de revistas específicas de endocrinologia. Engenharia e a Biologia também se destacaram no interesse em desenvolver e validar equipamentos de avaliação da reação motora, visto sua ampla aplicabilidade e para torná-los mais eficientes, de baixo custo e úteis para o público estudado. Ma et al. (2020) propõe, por exemplo, a investigação de um pedal de freio mais adaptado às mudanças de TR em diabéticos, a fim de melhorar sua performance e segurança no trânsito em função de um TF aumentado. 
Nesse sentido, percebe-se a necessidade da adaptação ao paciente com diabetes às atividades em geral, a fim de garantir sua segurança e melhora no desempenho, ampliando suas possibilidades terapêuticas.

Devido à ampla variedade de TR e formas de obtê-lo, não foi encontrada uma padronização metodológica ou equipamento que pudesse ser considerado "padrão-ouro" nesses estudos. A progressão das tecnologias é evidenciada, desde instrumentos simples com medidas únicas, até instrumentos elaborados que proporcionam dados mais precisos e a simulação de ambientes, a exemplo do TF. Os instrumentos mais utilizados foram aqueles disponíveis para aquisição no mercado e já utilizados em estudos anteriores. Os principais equipamentos comerciais foram PC $1000 \mathrm{~Hz}$ (Muhil, 2014), Anand Agencies Reaction Timer (Khode et al., 2015; Kour et al., 2019), M400 STISIM Drive Software (Ma et al., 2020), Stationary Simple Reaction Timer by Vericom Computers (Meyr \& Spiess, 2017; Sansosti et al., 2017; Spiess et al., 2017), Response Box by Eprime (Redondo et al., 2016), RTM 608 by Medicaid System (Mungal et al., 2015) e Slip-Falls plataform (Richerson et al., 2005). A vantagem observada no uso desses aparelhos comerciais foi o fato de serem validados e a maior confiabilidade interexaminador. Também apresentam um bom desempenho quando associados a medidas funcionais, como o tempo de frenagem. Já os aparelhos desenvolvidos pelos pesquisadores apresentam menor custo.

Sugere-se para estudos futuros, a adoção de medidas de limiar de percepção atual (current perception threshold) capazes de revelar o estado funcional dos distintos tipos de fibra nervosa (Inceu \& Veresiu, 2015), como forma de incrementar a interpretação de fatores associados à baixa velocidade de resposta motora nos pacientes com diabetes e identificar os danos em fases precoces.

\section{Conclusão}

A revisão integrativa identificou prejuízos devido a alteração do tempo de resposta motora no diabetes tipo 2 comparados a pessoas hígidas, com predomínio entre adultos, com doença crônica, sendo o tempo de resposta visual mais lento que o auditivo, e ambos afetados por condições associadas à cognição. As respostas motoras das funções manuais e pedais foram afetadas, afetando ações como dirigir ou condições de equilíbrio e postura, implicando na segurança e independência deste grupo de pessoas.

Em vista da predominância de estudos internacionais, foi observada a necessidade investigação da aplicabilidade desses instrumentos na população brasileira, a fim de validar seu uso no território considerando suas particularidades e epidemiologia. Também se sugere a padronização desses equipamentos na busca de um padrão-ouro, cujo procedimento possa ser replicado com base em evidências. Além disso, a associação com outras tecnologias, como as medidas de limiar de percepção atual, pode ampliar a interpretação das medidas encontradas e orientar o desenvolvimento de trabalhos futuros.

\section{Referências}

Almeida-Pititto, B. de, Almada Filho, C. de M., \& Cendoroglo, M. S. (2008). Déficit cognitivo: mais uma complicação do diabetes melito? . In Arquivos Brasileiros de Endocrinologia \& Metabologia (Vol. 52, pp. 1076-1083). scielo .

American Diabetes Association. (2012). Diagnosis and Classification of Diabetes Mellitus. Diabetes Care, 35(Supplement_1), S64-S71. https://doi.org/10.2337/dc12-s064

Botelho, L. L. R., de Almeida Cunha, C. C., \& Macedo, M. (2011). O método da revisão integrativa nos estudos organizacionais. Gestão e sociedade, 5(11), 121-136.

Ceriello, A., Monnier, L., \& Owens, D. (2019). Glycaemic variability in diabetes: clinical and therapeutic implications. The Lancet. Diabetes \& Endocrinology, 7(3), 221-230. https://doi.org/10.1016/S2213-8587(18)30136-0

Crocetta, T. B., Liz, C. M. de, \& Andrade, A. (2014). Características dos softwares de tempo de reação para mensurar respostas motoras: revisão sistemática. Revista Mineira de Educação Física, 22(3 SE-), 20-51. https://periodicos.ufv.br/revminef/article/view/10039

Dobrzański, T., \& Rychta, T. (1968). Studies on the time of simple reaction and selective reaction in diabetes and in schizophrenia. Polish Medical Journal, $7(2), 442-448$. 
Gilchrist, M., Winyard, P. G., Fulford, J., Anning, C., Shore, A. C., \& Benjamin, N. (2014). Dietary nitrate supplementation improves reaction time in type 2 diabetes: Development and application of a novel nitrate-depleted beetroot juice placebo. Nitric Oxide - Biology and Chemistry, 40, 67-74. https://doi.org/10.1016/j.niox.2014.05.003

Inceu, G. V., \& Veresiu, I. A. (2015). Measurement of current perception thresholds using the Neurometer(®) - applicability in diabetic neuropathy. Clujul Medical (1957), 88(4), 449-452. https://doi.org/10.15386/cjmed-491

Khode, V., Sindhur, J., Ramdurg, S., Ruikar, K., \& Nallulwar, S. (2015). Chronoscopic reading in whole body reaction times can be a tool in detecting cognitive dysfunction in type 2 diabetics: A case control study. JMS - Journal of Medical Society, 29(2), 69-73. https://doi.org/10.4103/0972-4958.163188

Kirkman, M. S., Briscoe, V. J., Clark, N., Florez, H., Haas, L. B., Halter, J. B., Huang, E. S., Korytkowski, M. T., Munshi, M. N., Odegard, P. S., Pratley, R. E., \& Swift, C. S. (2012). Diabetes in older adults. Diabetes Care, 35(12), 2650-2664. https://doi.org/10.2337/dc12-1801

Kour, H., Kothiwale, V. A., \& Goudar, S. S. (2019). Effects of the six months of programmed exercise therapy on cardio-respiratory endurance and neurophysiological variables in asymptomatic young adults diagnosed newly with type 2 diabetes mellitus - a randomized controlled trial. Indian Journal of Physiology and Pharmacology, 63(4), 283-293.

Ma, S., Zhang, J., Zeng, X., Wu, C., Zhao, G., Lv, C., \& Sun, X. (2020). Type 2 diabetes can undermine driving performance of middle-aged male drivers through its deterioration of perceptual and cognitive functions. Accident Analysis \& Prevention, 134, 105334. https://doi.org/https://doi.org/10.1016/j.aap.2019.105334

Meyr, A. J., \& Spiess, K. E. (2017). Diabetic Driving Studies-Part 1: Brake Response Time in Diabetic Drivers With Lower Extremity Neuropathy. The Journal of Foot and Ankle Surgery: Official Publication of the American College of Foot and Ankle Surgeons, 56(3), 568-572. https://doi.org/10.1053/j.jfas.2017.01.042

Ministério da Saúde do Brasil. (2020). Vigitel Brasil 2019: principais resultados. Boletim Epidemiológico, 51(16), $20-26$.

Moheet, A., Mangia, S., \& Seaquist, E. R. (2015). Impact of diabetes on cognitive function and brain structure. Annals of the New York Academy of Sciences, 1353, 60-71. https://doi.org/10.1111/nyas.12807

Muhil, M. (2014). Study of Auditory, Visual Reaction Time and Glycemic Control ( H BA 1 C ) in Chronic Type I I Diabetes Mellitus. Journal Of Clinical And Diagnostic Research, 134(November 2019), 105334. https://doi.org/10.7860/JCDR/2014/8906.4865

Mungal, S. U., Dube, S., \& Kulkarni, M. B. (2015). Comparative study of audiovisual reaction time in patients with type 2 diabetes mellitus and in normal subjects. National Journal of Physiology, Pharmacy and Pharmacology, 5(1), 54-55. https://doi.org/10.5455/njppp.2015.5.300720141

Pageaux, B., \& Lepers, R. (2018). The effects of mental fatigue on sport-related performance. Progress in Brain Research, 240, 291-315. https://doi.org/10.1016/bs.pbr.2018.10.004

Redondo, M. T., Beltrán-Brotóns, J. L., Reales, J. M., \& Ballesteros, S. (2016). Executive functions in patients with Alzheimer's disease, type 2 diabetes mellitus patients and cognitively healthy older adults. Experimental Gerontology, 83, 47-55. https://doi.org/10.1016/j.exger.2016.07.013

Richardson, J. K., Eckner, J. T., Allet, L., Kim, H., \& Ashton-Miller, J. A. (2017). Complex and Simple Clinical Reaction Times Are Associated with Gait, Balance, and Major Fall Injury in Older Subjects with Diabetic Peripheral Neuropathy. American Journal of Physical Medicine and Rehabilitation, 96(1), 816. https://doi.org/10.1097/PHM.0000000000000604

Richerson, S. J., Robinson, C. J., \& Shum, J. (2005). A comparative study of reaction times between type II diabetics and non-diabetics. Biomedical Engineering Online, 4, 12. https://doi.org/10.1186/1475-925X-4-12

Ryan, C. M., van Duinkerken, E., \& Rosano, C. (2016). Neurocognitive consequences of diabetes. The American Psychologist, 71(7), 563-576. https://doi.org/10.1037/a0040455

Sanchez-Marin, F. J., \& Padilla-Medina, J. A. (2010). Simple reaction times and performance in the detection of visual stimuli of patients with diabetes. Computers in Biology and Medicine, 40(6), 591-596. https://doi.org/10.1016/j.compbiomed.2010.04.003

Sansosti, L. E., Spiess, K. E., \& Meyr, A. J. (2017). Diabetic Driving Studies-Part 3: A Comparison of Mean Brake Response Time Between Neuropathic Diabetic Drivers With and Without Foot Pathology. The Journal of Foot and Ankle Surgery: Official Publication of the American College of Foot and Ankle Surgeons, 56(3), 577-580. https://doi.org/10.1053/j.jfas.2017.01.044

Sociedade Brasileira de Diabetes, Sociedade Brasileira de Patologia Clínica, \& Sociedade Brasileira de Endocrinologia e Metabologia. (2017). Atualização sobre Hemoglobina Glicada (A1C) para Avaliação do Controle Glicêmico e para o Diagnóstico do Diabetes: Aspectos clínicos e Laboratoriais. In Posicionamento Oficial SBD, SBPC-ML, SBEM e FENAD 2017/2018. https://doi.org/10.1590/S1676-24442009000100007

Spiess, K. E., Sansosti, L. E., \& Meyr, A. J. (2017). Diabetic Driving Studies-Part 2: A Comparison of Brake Response Time Between Drivers With Diabetes With and Without Lower Extremity Sensorimotor Neuropathy. The Journal of Foot and Ankle Surgery: Official Publication of the American College of Foot and Ankle Surgeons, 56(3), 573-576. https://doi.org/10.1053/j.jfas.2017.01.043

Whitehead, B. P., Dixon, R. A., Hultsch, D. F., \& MacDonald, S. W. S. (2011). Are neurocognitive speed and inconsistency similarly affected in type 2 diabetes? Journal of Clinical and Experimental Neuropsychology, 33(6), 647-657. https://doi.org/10.1080/13803395.2010.547845

World Health Organization. (n.d.). HEARTS D: diagnosis and management of type 2 diabetes. World Health Organization. https://apps.who.int/iris/handle/10665/331710

World Health Organization. (2016). Global report on diabetes. World Health Organization. https://apps.who.int/iris/handle/10665/204871

World Health Organization. (2019). Classification of diabetes mellitus. World Health Organization. https://apps.who.int/iris/handle/10665/325182

World Health Organization, \& International Diabetes Federal. (n.d.). Definition and diagnosis of diabetes mellitus and intermediate hyperglycaemia : report of a WHO/IDF consultation. World Health Organization. https://apps.who.int/iris/handle/10665/43588 